\title{
Use of the T-SPOT.TB Assay to Detect Latent Tuberculosis Infection Among Rheumatic Disease Patients on Immunosuppressive Therapy
}

\author{
SAMUEL M. BEHAR, DANIEL S. SHIN, AGNES MAIER, JONATHAN COBLYN, SIMON HELFGOTT, \\ and MICHAEL E. WEINBLATT
}

\begin{abstract}
Objective. We evaluated the T-SPOT.TB assay to identify latent tuberculosis infection (LTBI) in patients with rheumatic disease receiving immunosuppressive medication including tumor necrosis factor (TNF) antagonists.

Methods. A total of 200 patients seen in the Arthritis Center at Brigham and Women's Hospital were enrolled for study. Most patients were US-born women with rheumatoid arthritis. A medical history was obtained using a questionnaire, whole blood was drawn for the T-SPOT.TB assay, and tuberculin skin testing (TST) was performed.

Results. Both tests were performed on 179 subjects, who had no history of a positive TST. All subjects had a strong response to the T-SPOT.TB test positive control, and there were no indeterminate results. Among these 179 subjects, 2 had a positive TST and 10 had a positive T-SPOT.TB test. No subject was positive for both tests. Patients with a positive T-SPOT.TB test did not have typical risk factors for LTBI based on clinical evaluation.

Conclusion. The lack of concordance between the TST and the T-SPOT.TB assay may indicate that the immunoassay is more sensitive, particularly in a patient population taking immunosuppressive medications. It is equally likely that the low prevalence of LTBI in this low-risk population led to an increase in the false-positive rate despite the high sensitivity and specificity of the T-SPOT.TB assay. In the context of our patient population, the T-SPOT.TB assay is likely to be most useful in evaluation of patients with a positive TST, since these patients have a higher pretest probability of having LTBI. (First Release Feb 15 2009; J Rheumatol 2009;36:546-51; doi:10.3899/jrheum.08054)
\end{abstract}

Key Indexing Terms:

RHEUMATIC DISEASE TUMOR NECROSIS FACTOR ANTAGONISTS TIAGNOSIS

TUBERCULOSIS

ELISPOT

Tumor necrosis factor (TNF) antagonists have emerged as an important treatment for rheumatoid arthritis (RA) and other rheumatic diseases. However, their use is complicated by adverse effects including an increased incidence of infection. Several of the infections observed in patients undergoing TNF blockade are different from those usually observed in RA: patients with RA receiving TNF blockade have a greater risk of developing tuberculosis (TB) than the general public ${ }^{1-4}$. Surveillance studies found that the risk of developing TB among patients with RA receiving TNF antagonists was significantly higher than for RA patients

From the Division of Rheumatology, Immunology, and Allergy, Brigham and Women's Hospital, and Harvard Medical School, Boston,

Massachusetts, USA.

Supported by Oxford Immunotec, Ltd., Abingdon, Oxfordshire, UK.

S.M. Behar, MD, PhD; D.S. Shin, BS; A. Maier, BA; J. Coblyn, MD;

S. Helfgott, MD; M.E. Weinblatt, MD.

Address reprint requests to Dr. S.M. Behar, Brigham and Women's

Hospital, Smith Building, 5th floor, One Jimmy Fund Way, Boston, MA

02492.E-mail: sbehar@rics.bwh.harvard.edu

Accepted for publication October 31, 2008. receiving other therapies ${ }^{3-5}$. This risk is now described in the product labeling for all anti-TNF agents ${ }^{6}$.

TNF is critical for immunity to TB at all stages of infection. Anti-TNF treatment of mice chronically infected with $M$. tuberculosis leads to rapid recrudescence and death. Indeed, the development of TB during the first few months of TNF antagonist therapy is consistent with an accelerated transition from latent to reactivation disease ${ }^{1}$. In addition to inducing reactivation, RA patients receiving TNF antagonists may also be at greater risk for the development of primary TB.

Latent TB infection (LTBI) can be treated with prophylactic antibiotic therapy, which reduces the future risk of developing $\mathrm{TB}^{7}$. Therefore, patients should be screened for the presence of LTBI before starting TNF antagonists. Traditionally, the tuberculin skin test (TST) using purified protein derivative (PPD) has been used to identify cases of LTBI. However, the TST does not have high enough sensitivity or specificity (in populations that are vaccinated with BCG) to serve as an adequate diagnostic test ${ }^{8}$. Patients with RA pose an additional challenge because of the increased

Personal non-commercial use only. The Journal of Rheumatology Copyright @ 2009 . All rights reserved. 
prevalence of skin-test anergy due to intrinsic immune dysfunction and the effect of medications 9 .

A new generation of clinical immunoassays has been developed to diagnose active TB and LTBI ${ }^{10}$. These tests work by detecting $\gamma$-interferon (IFN- $\gamma$ ) production by T cells after stimulation with antigens specific for M. tuberculosis. The antigens used in these tests, CFP10 and ESAT-6, are expressed by M. tuberculosis but not by BCG. This property allows the assays to discriminate between BCG-vaccinated people and persons infected with M. tuberculosis. These tests are referred to as IFN- $\gamma$-release assays (IGRA) and 2 commercial formats are available. In the QuanteFERON Gold assay, M. tuberculosis antigens are added to whole blood, and the IFN- $\gamma$ in plasma is measured by ELISA the next day ${ }^{11}$. In contrast, the T-SPOT.TB assay is performed using isolated peripheral blood mononuclear cells (PBMC), and IFN- $\gamma$ production is detected using an Elispot assay ${ }^{11}$. The extra step of isolating PBMC has the effect of normalizing differences in the peripheral white blood cells, which may explain why the T-SPOT.TB test performs better in subjects with compromised immune systems including human immunodeficiency virus (HIV)-positive individuals ${ }^{12,13}$. We sought to determine whether the T-SPOT.TB test would be useful in assessing the risk of patients with RA who are taking immunosuppressive medication for LTBI.

\section{MATERIALS AND METHODS}

Patients. Between July 2006 and December 2006, 200 patients with a systemic rheumatic disease (RA, psoriatic arthritis, systemic lupus, vasculitis, polymyositis, dermatomyositis, sarcoidosis, polymyalgia rheumatica, ankylosing spondylitis, or scleroderma) were recruited to the study. These patients were undergoing systemic immunosuppressive therapies including methotrexate, cyclophosphamide, corticosteroids, azathioprine, abatacept, anakinra, rituximab, or anti-TNF therapies (etanercept, adalimumab, or infliximab). Patients were excluded from the study if they refused to give written consent or were currently undergoing therapy for TB.

Each patient was administered a questionnaire that addressed possible TB epidemiologic risk factors. Data collection included age, sex, ethnicity, country of birth and residence, contact and occupational exposure to TB, BCG vaccination status, family and personal history of mycobacterial infection, risk factors for TB (described below), and clinical information (including results of previous TST and chest radiographs). Risk factors for LTBI were considered to be (1) birth or resident for $>6$ months in a country with a TB prevalence $>20$ cases/100,000; (2) household contact; (3) chest radiographs showing calcified granulomas or apical densities; or (4) confirmed history of $\mathrm{TB}^{8}$. Other clinical information was obtained by review of the medical record.

All patients gave informed consent before enrollment in the study and the study was approved by the hospital institutional review board

Skin testing: The TST was done using PPD. A clinic nurse trained in Mantoux skin testing administered the test. Two tuberculin units were planted intradermally on the volar surface of the forearm using the Mantoux method. Patients were instructed and provided a measuring device to read their own results and telephone it in to the nurse 48 hours after the test. All patients reporting an abnormal TST result were asked to return to the Arthritis Clinic to have the reading confirmed by a nurse or physician. Induration $>5 \mathrm{~mm}$ was considered positive in this patient population. Some patients that had a previously documented positive TST were enrolled in the study but the TST skin test was not repeated in this group.
Elispot. At the same visit, blood was obtained for the T-SPOT.TB Elispot assay. The blood collection was performed the same day that the Mantoux test was placed. The T-SPOT.TB assay was performed using the T-SPOT.TB assay kit according to the manufacturer's recommendations. Briefly, $8 \mathrm{ml}$ blood was drawn into a BD vacutainer CPT $^{\mathrm{TM}}$ cell preparation tube with sodium citrate (BD Biosciences, Franklin Lakes, NJ, USA). The blood was processed within $4 \mathrm{~h}$. PBMC were isolated by centrifugation, and the cells were counted and resuspended in Aim V (Gibco/Invitrogen Corp., Gaithersburg, MD, USA) media at a concentration of $2.5 \times 10^{6} / \mathrm{ml} .100 \mu \mathrm{l}$ of the cell suspension was added to each of 4 wells that were precoated with anti-IFN- $\gamma$ antibody. The cells were cultured with antigen A (ESAT-6), antigen B (CFP10), a nil control, or a positive control (PHA) overnight at $37^{\circ} \mathrm{C}$ in $5 \% \mathrm{CO}_{2}$. After $16 \mathrm{~h}$, the wells were washed with phosphate buffered saline (PBS) and then incubated with conjugate reagent for $1 \mathrm{~h}$ at $2^{\circ}-8^{\circ} \mathrm{C}$. The plate was washed again with PBS and substrate solution was added. The spots were developed for $7 \mathrm{~min}$. The reaction was stopped by washing with distilled water. When the wells had dried, the spots were counted using a dissecting microscope. The manufacturer independently confirmed the number of spot counts. Participants, nurses, and physicians were blinded to the results of the T-SPOT.TB assay. Laboratory staff performing the TSPOT.TB assays were blinded to the results of the Mantoux test and patient data. Reactive tests were determined based on the following criteria as provided by the manufacturer: (1) when the nil control had fewer than 6 spots, and the "A" or "B" antigen spot count minus the nil control was $\geq 6$; (2) when the nil control had 6-10 spots, and the "A" or " $B$ " antigen spot count was $\geq 2 \times$ nil spot count; or (3) when the nil control had 11-20 spots, and the "A" or "B" antigen spot count was $\geq 3 \times$ nil spot count.

Statistics. All analysis was done using Prism 5.0 (GraphPad, San Diego, CA, USA). The kappa statistic for concordance was calculated using an online calculator (available from: http://www.graphpad.com/quickcalcs/ kappa1.cfm).

\section{RESULTS}

Comparison of the TST and T-SPOT.TB assay in subjects without a prior positive TST. The majority of the 200 patients had RA, with the remainder having psoriatic arthritis or other rheumatic diseases (Table 1). Patients were receiving anti-TNF blockade (including etanercept, adalimumab, or infliximab; 50.3\%), a disease-modifying

Table 1. Patient characteristic.

\begin{tabular}{lcc}
\hline Characteristic & $\mathrm{n}$ \\
\hline Age, yrs & $57 \pm 14$ & 191 \\
Female, \% & 74.3 & 142 \\
Caucasian, \% & 92.1 & 176 \\
US-born, \% & 88.5 & 169 \\
BCG vaccination, \% & 4.7 & 9 \\
Underlying disease, $\%$ & & \\
RA & 80.1 & 153 \\
PsA & 5.8 & 11 \\
Other/not reported & 8.9 & 17 \\
Other rheumatic disease & 5.2 & 10 \\
Treatment, \% & & \\
MTX \pm CS & 27.7 & 53 \\
TNF antagonists \pm CS & 25.7 & 49 \\
DMARD + TNF antagonists \pm CS & 24.6 & 47 \\
Other DMARD or biologic & 18.3 & 35 \\
\hline
\end{tabular}

RA: rheumatoid arthritis; PsA: psoriatic arthritis; MTX: methotrexate; CS: corticosteroid; TNF: tumor necrosis factor; DMARD: disease modifying antirheumatic drug.

Personal non-commercial use only. The Journal of Rheumatology Copyright (C) 2009. All rights reserved. 


\section{T-Spot.TB negative}

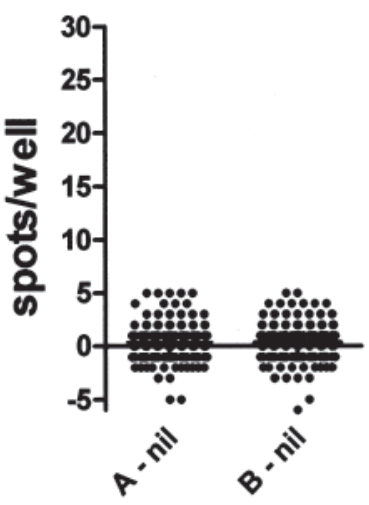

T-Spot.TB positive

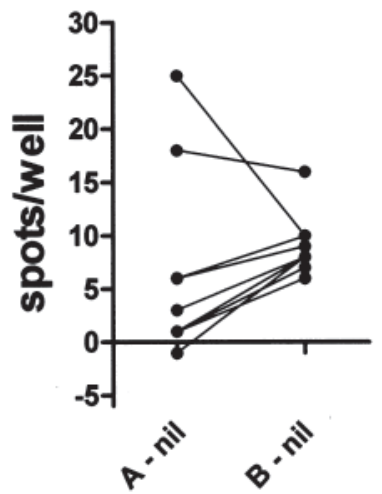

\section{Prior TST \\ positive}

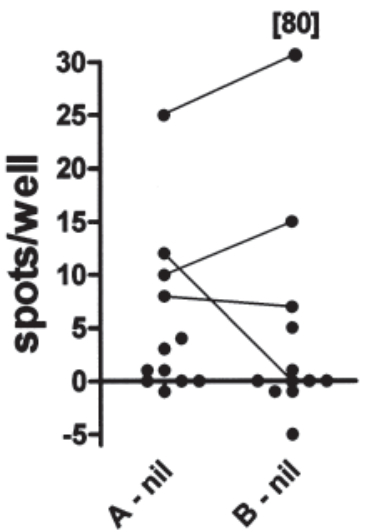

Figure 1. T-SPOT.TB test results are shown for each subject. The spot count for the "nil" control has been subtracted from the spot count for the "A" (ESAT6) and "B" (CFP10) antigens. Subjects with no history of positive TST are grouped according to their T-SPOT.TB test result, either negative (left panel) or positive (center panel). T-SPOT.TB results for the 12 subjects with a prior positive TST are also shown (right panel). Positive results from the same subject are connected by a line.

antirheumatic drug (methotrexate, azathioprine, cyclophosphamide, or leflunomide; 64\%), or another biologic agent (rituximab, abatacept, or anakinra; 7.3\%). Corticosteroid use was reported by $30 \%$ of the subjects. The other characteristics of the patient population are listed in Table 1. Nine subjects were excluded from the analysis because results of the TST could not be obtained $(\mathrm{n}=3)$ or the T-Spot.TB assay could not be performed $(n=6)$.

It was found that 179 subjects reported never having a prior positive TST, and had both a TST and a T-SPOT.TB assay performed as part of this study. Two subjects were found to have a positive TST, both with $>15 \mathrm{~mm}$ induration (Table 2); neither reported a history of BCG vaccination. One subject lived in Ireland until age 27 years, when she emigrated to the US in 1963; she works as a healthcare provider and reports a workplace contact. The other subject served in the military for 12 years including 6 years in Korea. Their chest radiographs were negative, and both individuals had nonreactive T-SPOT.TB assay results (Figure 1, Table 2).

Table 2. Previously TST-negative subjects found to have a positive TST or T-SPOT.TB test.

\begin{tabular}{|c|c|c|c|c|c|c|c|c|c|c|c|}
\hline \multirow[t]{2}{*}{ Age/Sex } & \multirow[t]{2}{*}{ Diagnosis } & \multirow{2}{*}{$\begin{array}{l}\text { Country } \\
\text { of Birth }\end{array}$} & \multirow[t]{2}{*}{ TB Risk Factors } & \multirow[t]{2}{*}{ Anti-TNF } & \multirow{2}{*}{$\begin{array}{l}\text { History of } \\
\text { BCG } \\
\text { Vaccination }\end{array}$} & \multicolumn{4}{|c|}{ T-Spot.TB* } & \multirow[t]{2}{*}{$\mathrm{TST}^{* *}$} & \multirow[t]{2}{*}{ Radiology $y^{\dagger}$} \\
\hline & & & & & & Nil & A & $\mathrm{B}$ & Result & & \\
\hline $47 \mathrm{~F}$ & PsA & USA & $\begin{array}{c}\text { Military 1979-91 } \\
\text { Korea, 1982-88, } \\
\text { multiple TST }\end{array}$ & None & No & 7 & 6 & 11 & NR & $>15 \mathrm{~mm}$ & Negative \\
\hline $59 \mathrm{M}$ & RA & USA & $\begin{array}{c}\text { Healthcare provider, } \\
\text { multiple TST }\end{array}$ & 2006 & No & 3 & 4 & 10 & $\mathrm{R}$ & NR & ND \\
\hline $47 \mathrm{~F}$ & RA & USA & Malta, 1992-94 & 1998 & No & 11 & 36 & 21 & $\mathrm{R}$ & NR & ND \\
\hline $58 \mathrm{~F}$ & RA & USA & & None & No & 0 & 6 & 10 & $\mathrm{R}$ & NR & \\
\hline $46 \mathrm{M}$ & PsA & USA & & 2005 & No & 2 & 20 & 18 & $\mathrm{R}$ & NR & ND \\
\hline $56 \mathrm{M}$ & RA & USA & & 2003 & No & 6 & 12 & 15 & $\mathrm{R}$ & NR & Negative \\
\hline $43 \mathrm{~F}$ & RA & USA & & 2002 & No & 1 & 2 & 7 & $\mathrm{R}$ & NR & Negative \\
\hline
\end{tabular}

V: vasculitis; PsA: psoriatic arthritis; RA: rheumatoid arthritis; NA: not available. * Elispot results expressed as spots/well (A, ESAT-6; B, CFP10); NR: not reactive; R: reactive. ${ }^{* *}$ Millimeters of induration; NR: not reactive $(<5 \mathrm{~mm}) .{ }^{\dagger}$ Chest radiograph results: negative: no disease present; ND: not documented. 
All subjects tested using the T-SPOT.TB assay had an adequate response to the control mitogen PHA, indicating that the $\mathrm{T}$ cells isolated from their peripheral blood were able to secrete IFN- $\gamma$ under the assay conditions (data not shown). Ten subjects had positive T-SPOT.TB assays (Table 2). Only 2 of these subjects had any significant risk of exposure to TB: one had worked as a healthcare provider for 40 years and another had lived in Malta for 3 years. None of the other 8 subjects with a positive T-SPOT.TB test reported risk factors associated with exposure to active cases of TB. Specifically, no one reported a history of TB, immigration from or travel within countries having a high TB burden, or close association with an active case of TB. Of note, 7 of these 10 patients were taking TNF antagonists ( 5 etanercept, 2 infliximab; see Table 2) for between 4 months and 11 years (median 4 yrs) with no evidence of active TB.

There was no overlap between the subjects with a positive TST and those with a positive T-SPOT.TB test. Although one of the subjects with a positive TST had 6 and 11 spots for the A and B antigens, the nil control was 7 spots, which is interpreted as negative by the manufacturer's criteria. The kappa statistic was -0.019 , indicating that agreement between the tests was worse than expected by chance; however, since no subject was positive by both tests, calculating the concordance was not meaningful.

Analysis of subjects with a prior positive TST. In addition to the 179 subjects described above, 12 additional subjects reported a prior positive TST. These 12 were tested by TSPOT.TB assay only and were analyzed separately (Figure 1, Table 3). In contrast to the other subjects, this group was more likely to be foreign-born (50\%) and to have received BCG vaccination (33\%). At least 4 of these subjects had exposure to cases of TB, and 4 had abnormal chest radio- graphs. Four subjects had positive T-SPOT.TB tests. Although all BCG-vaccinated subjects $(\mathrm{n}=4)$ had a negative T-SPOT.TB test, interpretation of this finding was confounded by the fact that all 4 had previously received isoniazid. Indeed, 10 of these 12 had received isoniazid in the past $(n=6)$ or were receiving prophylactic isoniazid therapy currently $(\mathrm{n}=4)$.

\section{DISCUSSION}

We evaluated the use of the T-SPOT.TB diagnostic assay to identify subjects that have LTBI and consequently may have an increased risk for development of active TB when treated with anti-TNF and other immunosuppressive medications. Subjects with rheumatic diseases treated with immunosuppressive medication were tested using the T-SPOT.TB blood test and a conventional TST. Among the subjects that had both a TST and a T-SPOT.TB test performed, all patients had a robust positive response to the PHA control, indicating that the immunosuppressive medications did not impair their immunological responses measured by this test. Ten patients were T-SPOT.TB-positive and TST-negative. Although these were exactly the patient subset that we had hoped to identify, 8 of these patients lacked clinical or radiological evidence to support the diagnosis of LTBI; therefore, we believe these T-SPOT.TB test results are most likely to be false-positives. Further, most of the subjects with a positive T-SPOT.TB test had been taking TNF antagonists for several years without evidence of clinical TB, and there had been no new cases since the tests were performed (duration of followup 15-19 mo). In most reported series, the majority of patients that developed active TB following treatment with TNF antagonists did so within a few months of starting therapy. Therefore, whether the

Table 3. T-SPOT.TB results in subjects with a history of a positive TST.

\begin{tabular}{|c|c|c|c|c|c|c|c|c|c|c|c|}
\hline \multirow[t]{2}{*}{ Age/Sex } & \multirow[t]{2}{*}{ Diagnosis } & \multirow{2}{*}{$\begin{array}{l}\text { Country } \\
\text { of Birth }\end{array}$} & \multirow[t]{2}{*}{ TB Risk Factors } & \multirow{2}{*}{$\begin{array}{l}\text { History of } \\
\text { BCG } \\
\text { Vaccination }\end{array}$} & \multicolumn{4}{|c|}{ T-Spot.TB* } & \multicolumn{2}{|c|}{$\mathrm{TST}^{* *}$} & \multirow{2}{*}{ Radiology $y^{\dagger}$} \\
\hline & & & & & Nil & A & $\mathrm{B}$ & Result & Year & Result & \\
\hline $59 \mathrm{~F}$ & RA & USA & Healthcare worker & No & 3 & 13 & 18 & $\mathrm{R}$ & 1971 & $10-14 \mathrm{~mm}$ & Negative \\
\hline $81 \mathrm{M}$ & RA & India & $\begin{array}{l}\text { Healthcare worker, } \\
\text { TB hospital }\end{array}$ & No & 2 & 14 & 2 & $\mathrm{R}$ & NA & $>15 \mathrm{~mm}$ & Interstitial fibrosis \\
\hline $57 \mathrm{~F}$ & PsA & USA & Father had active TB & No & 2 & 5 & 1 & NR & 1980 & $>15 \mathrm{~mm}$ & Negative \\
\hline $49 \mathrm{~F}$ & RA & Japan & & Yes & 8 & 7 & 3 & NR & NA & NA & Negative \\
\hline $60 \mathrm{~F}$ & RA & Peru & & No & 1 & 2 & 1 & NR & NA & $10-14 \mathrm{~mm}$ & Negative \\
\hline $25 \mathrm{~F}$ & RA & Korea & & Yes & 1 & 1 & 0 & NR & 2001 & NA & Granuloma; hilar Ca \\
\hline $40 \mathrm{~F}$ & RA & Japan & & Yes & 0 & 0 & 1 & NR & 2003 & $>15 \mathrm{~mm}$ & Negative \\
\hline $48 \mathrm{M}$ & RA & USA & & No & 0 & 1 & 0 & NR & 2002 & $10-14 \mathrm{~mm}$ & Negative \\
\hline
\end{tabular}

PsA: psoriatic arthritis; RA: rheumatoid arthritis. * Elispot results expressed as spots/well (A, ESAT-6; B, CFP10); NR: not reactive; R: reactive; Ca: calcification; RUL: right upper lung. $* *$ Millimeters of induration; NA: not available. ${ }^{\dagger}$ Chest radiograph results: negative: no disease present. ${ }^{\dagger \dagger}$ This subject with a previously normal chest radiograph was evaluated by bronchoscopy. Cultures were negative for M. tuberculosis. Her serologies were consistent with Chlamydia infection and appropriate treatment led to resolution of symptoms and chest radiograph normalized.

Personal non-commercial use only. The Journal of Rheumatology Copyright $\subset$ 2009. All rights reserved. 
T-SPOT.TB-positive tests represented false-positives or not, the test did not appear to be identifying subjects who were at increased risk for developing reactivation $\mathrm{TB}$ in our patient population.

There were several confounders in this study. The most important is that because of an increased prevalence of skin-test anergy among patients with RA, the TST may be unreliable. Patients receiving immunosuppressive or immunomodulatory medications may also have an altered response to skin testing, although whether cutaneous anergy in this population is an issue has not been definitively determined, as previously thought ${ }^{9,14,15}$. This may have been compounded by having the subjects self-read their TST. These factors may cause the TST to underestimate the true incidence of prior $M$. tuberculosis infection. In contrast, the T-SPOT.TB assay did not seem to be affected, in as much as the positive mitogen (PHA) control worked well in all individuals tested, suggesting that the ability of their $\mathrm{T}$ cells to secrete IFN- $\gamma$ under these conditions was preserved. Further, therapy with TNF antagonists did not appear to affect the test, since the frequency of positive tests was similar in patients receiving TNF antagonists and those not (10/96 vs 4/95, respectively; $p=0.163$, Fisher's exact test).

Although many studies have reported the superb specificity of the T-SPOT.TB test ${ }^{16}$, the positives in our population could also represent cross-reaction with other species of mycobacterium that have been described to infect this patient population ${ }^{17,18}$. Finally, although the results were independently confirmed, there is a subjective component to determining what is a "true" spot, and in a low-prevalence population, there can be a tendency to over-read the plates. Thus, in this outpatient population with a low pretest probability of LTBI, we observed a lack of concordance between the TST and the T-SPOT.TB.

A potentially important application of IGRA is their use to monitor the response to therapy. We evaluated 12 subjects with previously documented positive TST, of whom 10 had received prophylactic treatment with isoniazid. The frequency of antigen-specific $\mathrm{T}$ cells declines following treatment with antibiotics both in experimental animals and in humans, which represents contraction of clonally expanded $\mathrm{T}$ cells following antigen clearance ${ }^{19,20}$. Understanding this phenomenon in human subjects has important implications for monitoring treatment of $\mathrm{TB}$, particularly drug-resistant disease. Whether individuals with a positive T-SPOT.TB assay will convert to negative after successful treatment requires further study, since there are reports of persistently positive subjects after isoniazid prophylaxis as well as those that revert to negative ${ }^{21-23}$. Our data point to some problems inherent in this type of evaluation. Ten previously positive PPD+ subjects were treated with isoniazid, and 3 were still T-SPOT.TB-positive. Although these 3 subjects may have sustained memory immunity, it is also possible that they har- bor isoniazid-resistant TB. Thus, ascertaining the role of IGRA in monitoring therapy will require careful prospective immunological and microbiological studies in patients being treated for TB.

Patients receiving TNF antagonists require screening to identify those that may be latently infected with $M$. tuberculosis, and hence at increased risk for developing active $\mathrm{TB}^{24}$. We found that peripheral blood cells from patients with rheumatic disease receiving immunosuppressive medications all had a strong response to the mitogen-positive control in the TB-SPOT.TB assay. Among these participants, several had positive TB-SPOT.TB tests without historical or laboratory evidence classically associated with LTBI. Therefore, while there are many advantages of the T-SPOT.TB assay, more study of it and other IGRA is needed before these tests can be used for general screening of patients for $\mathrm{LTBI}^{25}$. In light of the recommendation by the US Centers for Disease Control that the TST can be replaced with IGRA ${ }^{26}$, it is interesting how different our results were with the 2 tests. Thus, although a recent study from Greece suggested that the TB-SPOT.TB test appeared useful in identifying patients with LTBI prior to initiation of TNF antagonists $^{27}$, in our clinical setting, the T-SPOT.TB assay may be most useful in further evaluation of patients with a positive TST, particularly in those individuals with a history of BCG vaccination who are from TB-endemic areas. Determining whether IGRA are useful for detecting LTBI will require longterm followup. Their use in subjects receiving TNF blockade is also complicated, since TNF antagonists have the potential to reduce IFN- $\gamma$ secretion by $M$. tuberculosisspecific $\mathrm{T}$ cells ${ }^{28}$. Finally, we believe that development of a clinical paradigm ${ }^{29}$ may be the most effective way to identify patients with LTBI who may have a higher risk for developing reactivation TB while on therapy for systemic rheumatic disease.

\section{REFERENCES}

1. Wallis RS, Broder M, Wong J, Beenhouwer D. Granulomatous infections due to tumor necrosis factor blockade: correction. Clin Infect Dis 2004;39:1254-5.

2 Keane J, Gershon S, Wise RP, et al. Tuberculosis associated with infliximab, a tumor necrosis factor- $\alpha$-neutralizing agent. N Engl J Med 2001;345:1098-104.

3. Bongartz T, Sutton AJ, Sweeting MJ, Buchan I, Matteson EL, Montori V. Anti-TNF antibody therapy in rheumatoid arthritis and the risk of serious infections and malignancies: systematic review and meta-analysis of rare harmful effects in randomized controlled trials. JAMA 2006;295:2275-85.

4. Wolfe F, Michaud K, Anderson J, Urbansky K. Tuberculosis infection in patients with rheumatoid arthritis and the effect of infliximab therapy. Arthritis Rheum 2004;50:372-9.

5. Gomez-Reino JJ, Carmona L, Valverde VR, Mola EM, Montero MD. Treatment of rheumatoid arthritis with tumor necrosis factor inhibitors may predispose to significant increase in tuberculosis risk: a multicenter active-surveillance report. Arthritis Rheum 2003;48:2122-7.

6. Food and Drug Administration. Arthritis Drugs Advisory Committee. Update on the TNF- $\alpha$ blocking agents. Rockville, MD:

Personal non-commercial use only. The Journal of Rheumatology Copyright @ 2009 . All rights reserved. 
Food and Drug Administration; 2003.

7. Horsburgh CR Jr. Priorities for the treatment of latent tuberculosis infection in the United States. N Engl J Med 2004;350:2060-7.

8. Diagnostic Standards and Classification of Tuberculosis in Adults and Children. This official statement of the American Thoracic Society and the Centers for Disease Control and Prevention was adopted by the ATS Board of Directors, July 1999. This statement was endorsed by the Council of the Infectious Disease Society of America, September 1999. Am J Respir Crit Care Med 2000;161:1376-95.

9. Greenberg JD, Reddy SM, Schloss SG, et al. Comparison of an in vitro tuberculosis interferon- $\gamma$ assay with delayed-type hypersensitivity testing for detection of latent Mycobacterium tuberculosis: A pilot study in rheumatoid arthritis. J Rheumatol 2008;35:770-5.

10. Lalvani A. Diagnosing tuberculosis infection in the 21 st century: new tools to tackle an old enemy. Chest 2007;131:1898-906.

11. Richeldi L. An update on the diagnosis of tuberculosis infection. Am J Respir Crit Care Med 2006;174:736-42.

12. Piana F, Codecasa LR, Cavallerio P, et al. Use of a T-cell-based test for detection of tuberculosis infection among immunocompromised patients. Eur Respir J 2006;28:31-4.

13. Rangaka MX, Wilkinson KA, Seldon R, et al. Effect of HIV-1 infection on T-cell-based and skin test detection of tuberculosis infection. Am J Respir Crit Care Med 2007;175:514-20.

14. Fuchs I, Avnon L, Freud T, Abu-Shakra M. Repeated tuberculin skin testing following therapy with TNF- $\alpha$ inhibitors. Clin Rheumatol 2009;28;167-72.

15. Ponce de Leon D, Acevedo-Vasquez E, Alvizuri S, et al. Comparison of an interferon- $\gamma$ assay with tuberculin skin testing for detection of tuberculosis (TB) infection in patients with rheumatoid arthritis in a TB-endemic population. J Rheumatol 2008;35:776-81.

16. Pai M, Zwerling A, Menzies D. Systematic review: T-cell-based assays for the diagnosis of latent tuberculosis infection: An update. Ann Intern Med 2008;149:177-84.

17. van Ingen J, Boeree M, Janssen M, et al. Pulmonary Mycobacterium szulgai infection and treatment in a patient receiving anti-tumor necrosis factor therapy. Nat Clin Pract Rheumatol 2007;3:414-9.

18. Salvana EM, Cooper GS, Salata RA. Mycobacterium other than tuberculosis (MOTT) infection: an emerging disease in infliximab-treated patients. J Infect 2007;55:484-7.
19. Kamath A, Woodworth JS, Behar SM. Antigen-specific CD8+ T cells and the development of central memory during Mycobacterium tuberculosis infection. J Immunol 2006;177:6361-9.

20. Dheda K, Pooran A, Pai M, et al. Interpretation of Mycobacterium tuberculosis antigen-specific IFN- $\gamma$ release assays (T-SPOT.TB) and factors that may modulate test results. J Infect 2007;55:169-73.

21. Pai M, Joshi R, Dogra S, et al. Persistently elevated T cell interferon-gamma responses after treatment for latent tuberculosis infection among health care workers in India: a preliminary report. J Occup Med Toxicol 2006;1:7.

22. Higuchi K, Harada N, Mori T. Interferon- $\gamma$ responses after isoniazid chemotherapy for latent tuberculosis. Respirology 2008;13:468-72.

23. Pai M, Joshi R, Bandyopadhyay M, et al. Sensitivity of a whole-blood interferon- $\gamma$ assay among patients with pulmonary tuberculosis and variations in T-cell responses during anti-tuberculosis treatment. Infection 2007;35:98-103.

24. Saag KG, Teng GG, Patkar NM, et al. American College of Rheumatology 2008 recommendations for the use of nonbiologic and biologic disease-modifying antirheumatic drugs in rheumatoid arthritis. Arthritis Rheum 2008;59:762-84.

25. Menzies D, Pai M, Comstock G. Meta-analysis: New tests for the diagnosis of latent tuberculosis infection: Areas of uncertainty and recommendations for research. Ann Intern Med 2007;146:340-54.

26. Mazurek GH, Jereb J, Lobue P, Iademarco MF, Metchock B, Vernon A. Guidelines for using the QuantiFERON-TB Gold test for detecting Mycobacterium tuberculosis infection, United States. MMWR Recomm Rep 2005;54:49-55.

27. Vassilopoulos D, Stamoulis N, Hadziyannis E, Archimandritis AJ. Usefulness of enzyme-linked immunospot assay (Elispot) compared to tuberculin skin testing for latent tuberculosis screening in rheumatic patients scheduled for anti-tumor necrosis factor treatment. J Rheumatol 2008;35:1271-6.

28. Hamdi H, Mariette X, Godot V, et al. Inhibition of anti-tuberculosis T-lymphocyte function with tumour necrosis factor antagonists. Arthritis Res Ther 2006;8:R114.

29. Catanzaro A, Perry S, Clarridge JE, et al. The role of clinical suspicion in evaluating a new diagnostic test for active tuberculosis: Results of a multicenter prospective trial. JAMA 2000;283:639-45. 